\title{
Digitalización del entorno a partir de un LIDAR HDL-64E
}

\author{
José de Jesús Rico Jimenez ${ }^{1}$, José Joel González Barbosa ${ }^{1}$, \\ Juan Bautista Hurtado Ramos ${ }^{1}$, Francisco Javier Ornelas Rodríguez, \\ Denis-Eduardo Hernandez Garcia ${ }^{1 *}$, Ricardo Gonzalez-Barbosa ${ }^{2}$ \\ ${ }^{1}$ Instituto Politécnico Nacional (CICATA-Querétaro), \\ Cerro Blanco 141, Col. Colinas del Cimatario, Querétaro, México \\ jgonzalezba@ipn.mx denis.hernandez@fcys.uni.edu.ni \\ **Universidad de Guanajuato, Campus Irapuato-Salamanca, México
}

(Recibido/received: 01-Octubre-2011; aceptado/accepted: 27-Febrero-2012)

\section{RESUMEN}

En este trabajo se propone un sistema de reconstrucción de mapas para un vehículo autónomo equipado con tecnología LIDAR, capaz de obtener más de un millón de puntos por segundo. Dentro del artículo proponemos la construcción rápida de Mapas Locales (ML) 2D que nos servirán para la navegación local autónoma, y la construcción de Mapas Globales (MG) 2D y 3D que modelizán el ambiente recorrido por el vehículo. Durante el proceso de construcción del Mapa Global se calcula la localización del vehículo con respecto a su posición inicial.

Palabras claves: Construcción de mapas 2D, construcción de mapas 3D, localización.

\begin{abstract}
This paper proposes a map building system for an autonomous vehicle equipped with LIDAR technology, capable to obtain more than one million points per second. This paper proposes a Fast Local Map building Approach (LM) that it is use for autonomous local navigation, and construction of Global Map (GM) 2D and 3D for modeling the whole environment crossed by the vehicle. During the process of the global map building, we estimate the location of the vehicle with respect to its initial position.
\end{abstract}

Keywords: 2D map building, 3D map building, localization.

\footnotetext{
* Autor para la correspondencia
} 


\section{INTRODUCCIÓN}

La tecnología LIDAR (Light Detection And Ranging ) ha sido utilizada en la localización de vehículos y construcción de mapas 3D por (Lingemann et al. (2005)), así como en reconstrucción 3D de ciudades ( $\mathrm{Pu}$ and Vosselman (2009); Arayici (2007)). Los sistemas de navegación autónoma necesitan de un modelo preciso del entorno que lo rodea con el propósito de llevar a cabo sus tareas de forma rápida, segura y exitosa. Sin este modelo, un robot no puede planear su ruta hacia su objetivo ni buscar un objeto o lugar. La reconstrucción 3D de ciudades es utilizada en proyectos arquitectónicos para remodelación y restauración, para promover sitios turísticos en los cuales se puede acceder virtualmente desde cualquier parte del mundo por medio de internet, para hacer un levantamiento de sitios arqueológicos, y para la toma decisiones en planeación urbana al recopilar información sobre avenidas, edificios, manzanas, transporte, etc.

El desarrollo de métodos de localización y mapeo simultáneos (SLAM) ha sido uno de los principales retos por varias décadas en el área de navegación autónoma para robots móviles (Gil A et at. (2010), Eade et al. (2009) ). Varios enfoques se han propuesto para la reconstrucción 3D de un entorno, haciendo uso de un robot móvil. En (Wangsiripitak et al. (2009)) se muestra un método de reconstrucción monoSLAM con un rastreador de objetos 3D, el proceso de SLAM provee al sistema dinámico información acerca de la trayectoria, movimiento y oclusión dentro de la construcción del mapa. El problema de SLAM es difícil por la siguiente paradoja: para moverse de manera precisa, un robot móvil debe tener un mapa del ambiente exacto; sin embargo, para construir un mapa preciso, las localizaciones del robot deben conocerse de forma muy exacta. El problema surge debido a los errores de odometría, en Caballero F. et al (2009) utilizan un odómetro visual integrado a SLAM para reducir la incertidumbre acumulada en la correspondencias de mapas, la incertidumbre es minimizada al fusionar varias adquisiciones provenientes de los sensores, la utilización de varios sensores es utilizada para mejorar la precisión en Schleicher D. et al.(2009) muestran una jerarquía topológica utilizando un sistema estéreo y un GPS dentro del sistema dinámico, se reconstruye un mapa secuencial 3D simultáneamente se conoce la actitud, localización y trayectoria del móvil. Se utilizan puntos característicos en el entorno para solventar las correspondencias de mapas y métodos bayesianos para modelar el comportamiento dinámico. Un análisis histórico y crítico de SLAM ha sido discutido en (Frese
(2006); Durran-Whyte and Bailey (2006); Baiely and Durran-Whyte (2006)).

Uno de los problemas más difíciles para cualquier algoritmo basado en el modelo de localización no es la extracción de características, sino la validación de éstas y la asociación de datos. Esto es para confirmar que la característica extraída es válida y asociarla con una característica conocida o estimada en el mapa del mundo. Los enfoques recientes hacen uso de la intensidad de láser para reconocer marcas del terreno; en Mullane et al. (2007) se presenta la caracterización del láser y los problemas de diseño para la detección de marcas del terreno con un tipo de láser. Esto demuestra que la localización de alta precisión se puede obtener con esta información, en N. Fioraio et al. (2011), hacen uso de cámaras con imágenes de profundidad tipo kinect para la reconstrucción del mapa que describe el entorno en aplicación de visual SLAM, este trabajo es el resultado del análisis y experimentación de (J. J. Rico Jiménez (2009)) en donde se realiza la reconstrucción de mapas, se encuentra el plano del suelo y otros planos presentes en la nube de puntos proporcionada por el HDL-64E.

\section{METODOLOGÍA}

El LIDAR HDL-64E (ver figura 1a) es un arreglo de sensores lásers de segunda generación que obtiene las distancias de los objetos situados a su alrededor. Este sensor ha sido diseñado para aplicaciones de navegación de vehículos autónomos, vigilancia estacionaria y móvil, construcción de mapas industriales y otras aplicaciones. El LIDAR tiene montado un arreglo de 64 láseres girando en el eje vertical ofreciendo un campo de visión horizontal de 360 grados. El campo de visión vertical es de 26.8 grados, que va desde $2^{\circ}$ hasta $-24.8^{\circ}$. Con un alcance de hasta 60 metros y un margen de error de \pm 5 $\mathrm{cm}$. El láser genera pulsos de 10 nanosegundos con una longitud de onda de 905 nanómetros.

La construcción de mapas se desarrolla en dos etapas (ver figura 2). La primera etapa presentada en la sección 3.1 genera un mapa 2D que permite estimar los objetos alrededor del auto, este mapa también es utilizado para encontrar la transformación rígida entre mapas consecutivos. La segunda etapa, presentada en la sección 3.2 , permite construir un mapa $2 \mathrm{D}$ y $3 \mathrm{D}$ de todo el ambiente en el cual se ha desplazado el automóvil. En esta misma etapa, el auto conoce la localización respecto a la posición donde se adquirió el primer mapa. 
Rico, J., et al

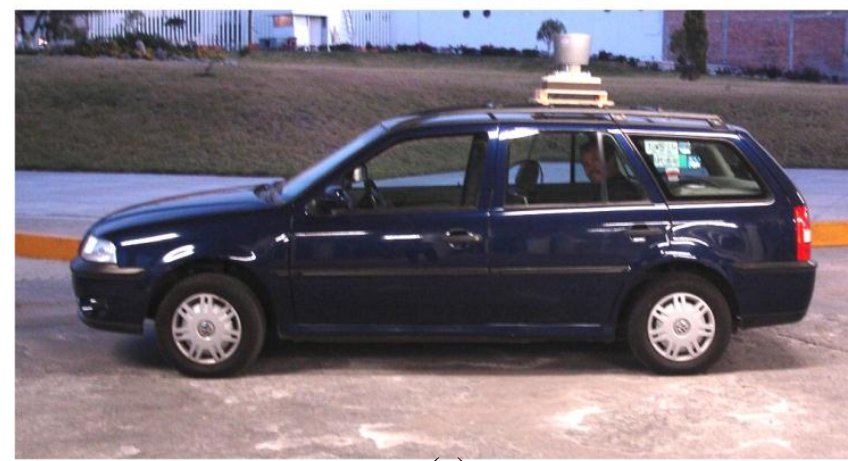

(a)

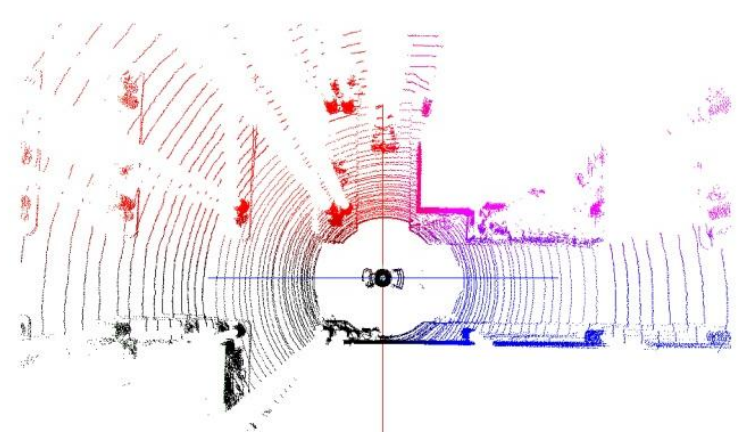

(b)

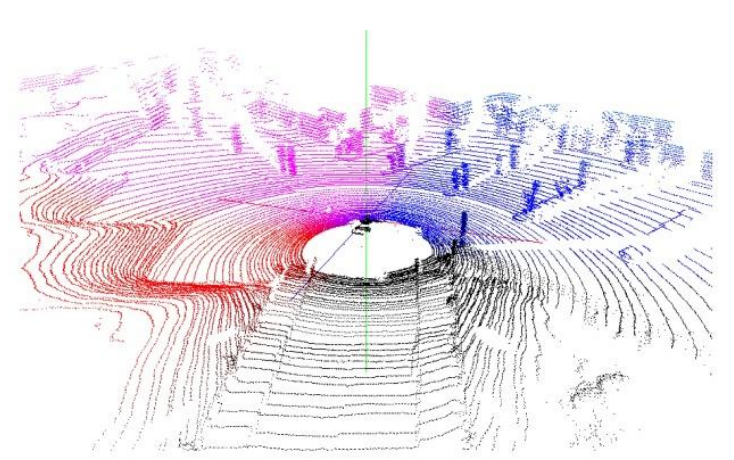

(c)

Figura 1. (a) LIDAR montado sobre el automóvil. El LIDAR está compuesto por 64 láseres. La orientación de cada láser está en función de $\phi$ y $\theta$. El ángulo $\phi$ es fijo, mientras que el ángulo $\theta$ está en función de la rotación del LIDAR. (b) y (c) ejemplos de nube de puntos.

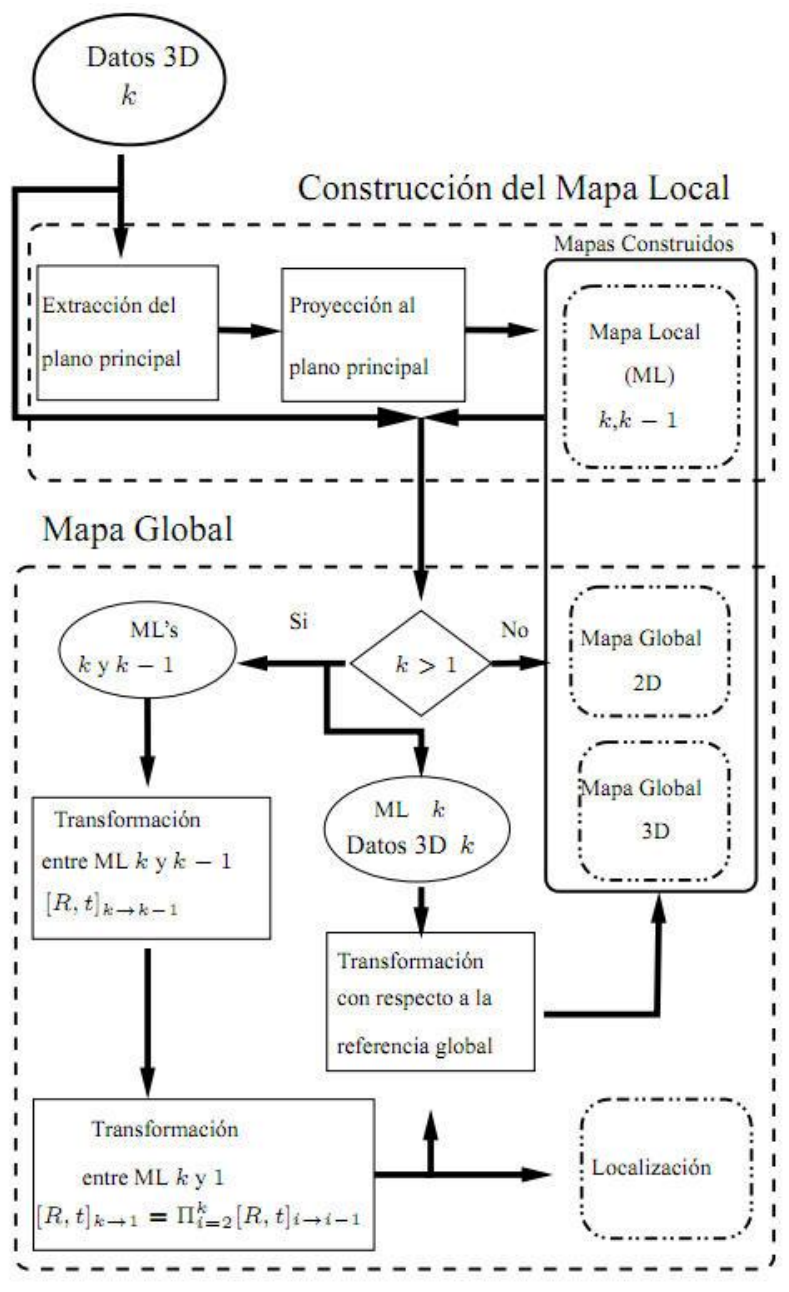

Figura 2. Diagrama de flujo de la construcción de mapas

\section{ENFOQUE MATEMÁTICO}

\section{Construción de mapas locales}

La construcción de mapas locales nos permite conocer obstáculos alrededor del automóvil y modelizar el ambiente localmente. Partiendo del conocimiento de que el ambiente es estructurado, se extrae de la nube de puntos 3D el plano principal, por el cual el automóvil puede desplazarse. El resto de puntos son proyectados sobre este plano para construir un mapa de navegación local. 
Rico, J., et al

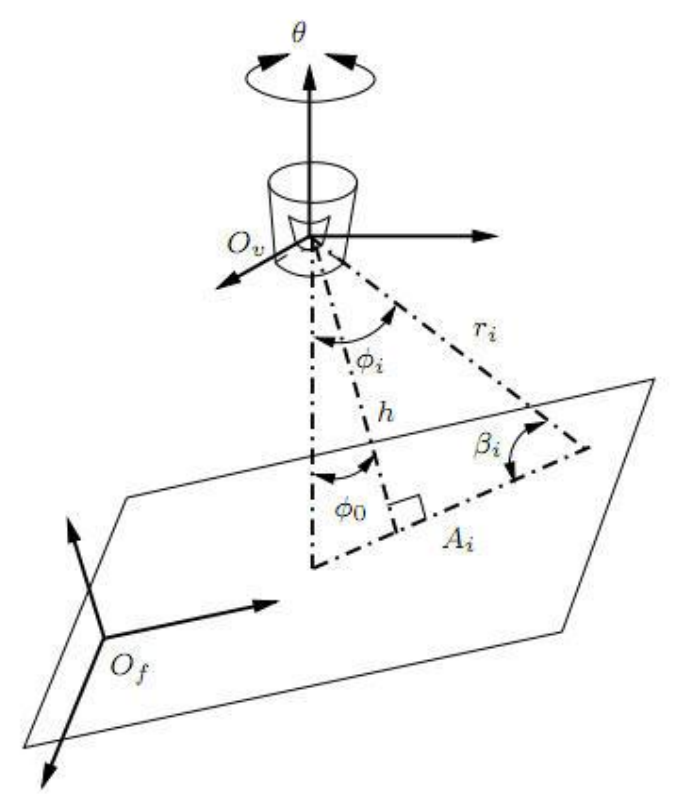

Figura 3. Para un ángulo $\theta$, el láser i forma un triángulo con el vector normal h del plano principal y la referencia $\mathrm{Z}$ del LIDAR.

\subsection{Extracción del plano principal}

El LIDAR es colocado sobre el automóvil (ver figura 1). La relación entre la referencia del LIDAR Ov y el plano principal Of se muestra en la figura 3. Para un ángulo de rotación de $\operatorname{LIDAR}(\theta)$, el láser i (donde $\mathrm{i}=1,2,3 \ldots$ 64) forma un triángulo con el plano del suelo y su normal $h$. Se puede expresar la relación de la figura 3 como:

$$
-h+r_{i} \cos \left(\phi_{i}\right) \cos \left(\phi_{0}\right)+r_{i} \sin \left(\phi_{i}\right) \sin \left(\phi_{0}\right)=0
$$

donde ri es la distancia entre el LIDAR a un objeto o plano principal en dirección $(\theta, \phi i)$ del láser $i, y ~ \phi 0$ corresponde a la orientación de la normal del plano principal respecto a eje $\mathrm{Z}$ del LIDAR. Por lo que la última ecuación puede escribirse por cada láser como:

$$
A x=0
$$

donde:

$$
A=\left[\begin{array}{ccc}
-1 & r_{1}(\theta) \cos \left(\phi_{1}\right) & r_{1}(\theta) \sin \left(\phi_{1}\right) \\
-1 & r_{2}(\theta) \cos \left(\phi_{2}\right) & r_{2}(\theta) \sin \left(\phi_{2}\right) \\
\cdot & \cdot & \cdot \\
\cdot & \cdot & \cdot \\
\cdot & \cdot & \cdot \\
-1 & r_{k}(\theta) \cos \left(\phi_{k}\right) & r_{k}(\theta) \sin \left(\phi_{k}\right)
\end{array}\right]
$$

$$
1 \leq k \leq 64, \quad x=\left[\begin{array}{c}
h \\
\cos \left(\phi_{0}\right) \\
\sin \left(\phi_{0}\right)
\end{array}\right]
$$

Si A $=$ ULVT es la descomposición de valores singulares de A, la solución de x está dada por vl, dode vl es el vector propio que corresponde al valor propio más pequeño $\sigma$ l diferente de cero.

La figura 3 muestra que la ecuación 1 es correcta cuando la normal $\mathrm{h}$ pertenece al plano formado por la dirección de los láseres. En cualquier otro caso, la ecuación 1 no puede ser usada para calcular la normal y el error de la solución de la ecuación se incrementa. Este error es el que usaremos para determinar los parámetros del plano.

\subsection{Mapa 2D}

Para la creación de un mapa local 2D se capturan los datos generados por el LIDAR en un giro de $360^{\circ}$. Es decir, como sólo interesa conocer las zonas que son navegables, que no tienen obstáculos. Una vez localizado el plano principal, la nube de puntos es rotada con el propósito de que el plano principal contenga los ejes $\mathrm{X}^{\prime}$ y $\mathrm{Y}^{\prime}$. A partir de esta información se define una cuadrícula del mapa que evidencia las características del ambiente. La cuadrícula del mapa es definida de la siguiente forma:

Una cuadrícula $\mathrm{M}$ es un arreglo bidimensional formado por una cuadrícula que modela o evidencia las características del ambiente. El mundo 3D es ortográficamente proyectado sobre un plano paralelo al plano principal que corresponde al suelo (asumiendo un ambiente estructurado donde la superficie del suelo corresponde a un plano). Debido a las características de reconstrucción, el centro del plano principal corresponde a la posición del LIDAR. Los puntos lejanos al centro son muy ruidosos por lo que definimos una región de interés (ROI) representada por un cuadrado de dimensiones $2 \Delta\left(-\Delta \leq \mathrm{x}^{\prime} \leq \Delta,-\Delta \leq \mathrm{y}^{\prime} \leq \Delta\right)$ sobre el plano 
principal $\left(\Psi X^{\prime} Y{ }^{\prime}\right)$. El plano es discretizado en cuadrículas (i, j) (donde $\mathrm{i}=1,2, \ldots \mathrm{m}, \mathrm{j}=1,2, \ldots \mathrm{n}$ ). Cada cuadrícula corresponde a un área rectangular (Aij ) del plano $\Psi \mathrm{X}^{\prime} \mathrm{Y}^{\prime}$. El área de intersección entre dos cuadrículas es siempre nula (Aij $\cap \mathrm{Akl}=\emptyset,(\mathrm{i}, \mathrm{j})=(\mathrm{k}$, 1)). Las áreas Aij no son necesariamente rectangulares y pueden no ser iguales. El subíndice i especifica la componente lateral y el $\mathrm{j}$ la componente de profundidad. Cada cuadrícula contiene una característica física del área de trabajo que representa, y dependiendo de la característica corresponde al mapa formado, estos pueden ser mapas binarios y mapas de intensidades.

Mapas binarios. Los puntos proyectados al plano principal y que pertenecen a la ROI son evaluados para determinar su posición en la cuadrícula (i, j), es decir:

$$
\begin{aligned}
& M_{I N}(i, j)=\#\left\{P\left(x^{\prime \prime}, y^{\prime \prime}\right) \mid \delta_{i}(i-1) \leq x^{\prime \prime} \leq \delta_{i}(i),\right. \\
& \left.\delta_{j}(j-1) \leq y^{\prime \prime} \leq \delta_{j}(j)\right\} \\
& \qquad P\left(x^{\prime \prime}, y^{\prime \prime}\right)=P\left(x^{\prime}, y^{\prime}\right)-[\Delta]^{T}, \delta_{i}=\frac{2 \Delta}{m} \\
& \text { donde: }
\end{aligned}
$$

$\mathrm{y}$

$$
\delta_{j}=\frac{2 \Delta}{n}
$$

En la adquisición existen puntos aislados que no pertenecen a ningún objeto u obstáculo, por lo que las zonas navegables se determinan de acuerdo a la siguiente ecuación:

$$
M_{B I}=\left\{\begin{array}{l}
1, M_{I N}(i, j) \geq \tau \\
0, M_{I N}(i, j)<\tau
\end{array}\right.
$$

donde 0 indican zonas navegables, mientras que 1 indica zonas de obstáculos. El valor de $\tau$ permite filtrar ruido de puntos 3D.

Mapas de intensidades. El Mapa de intensidades, (MINT ), se forma con los valores promedio de las intensidades de los lásers en una cuadrícula en especial. Este mapa está normalizado a una escala de intensidad de 0 a 255.

Cada punto 3D del LIDAR [P( $\left.\left.\mathrm{x}^{\prime \prime}, \mathrm{z}^{\prime \prime}, \mathrm{y}^{\prime \prime}\right)\right]$ contiene la información de intensidad del láser, por lo que cada punto puede ser descrito como $\mathrm{P}\left(\mathrm{x}^{\prime \prime}, \mathrm{z}^{\prime \prime}, \mathrm{y}^{\prime \prime}, \gamma\right)$. La intensidad del láser depende de varios factores, entre los más importantes es el tipo de material, color y textura. El mapa de intensidades es construido por el promedio de la intensidad $\gamma$ de los puntos que caen en la zona evaluada. La suma de las intensidades es definida como:

$$
T_{I N T}(i, j)=\sum_{\delta_{i}(i-1) \leq x^{\prime \prime} \leq \delta_{i}(i), \delta_{j}(j-1) \leq z^{\prime \prime} \leq \delta_{j}(j)} \gamma
$$

El mapa 2D formado por el promedio de las intensidades está definido de la siguiente forma :

$$
M_{I N T}(i, j)=\left\{\begin{array}{c}
\frac{T_{I N T}(i, j)}{M_{I N}(i, j)}, \text { siM } \\
0, \text { enotrocaso }
\end{array}\right.
$$

\section{Construcción del mapa global}

La adquisición de los puntos del LIDAR son referenciados respecto a la posición del LIDAR. Sin embargo, el LIDAR se encuentra en movimiento, por lo que cada nube de puntos tiene una referencia diferente. Una vez creados los mapas individuales se debe calcular la transformación rígida entre mapas consecutivos de manera secuencial. Calcular la transformación rígida entre los mapas consecutivos a partir de marcas en los mapas 3D es un problema complejo. Partiendo del conocimiento que el auto se desplaza en un ambiente estructurado, los mapas 3D son transformados a mapas 2D (como se explicó en la sección 3.2). A partir de los mapas 2D se extraen los puntos de interés de cada mapa de intensidades (MINT) con el detector de esquinas de (Harris and Stephens (1988)), se evalúa cuáles son comunes entre los dos mapas y se calcula una matriz de transformación rígida entre ellos.

Dado que para generar el mapa Global se necesita una sola referencia, en este trabajo se propone tomar la primer referencia del LIDAR como la referencia Global (ver figura 2). Por tal razón, cada nube de puntos (con referencia diferente) es transformada a la referencia del primer mapa. Para calcular la transformación homogénea del mapa $\mathrm{k}$ con respecto al primer mapa ([R, $\mathrm{t}] \mathrm{k} \rightarrow 1$ ), se calcula la transformación del mapa $\mathrm{k}$ a la referencia $\mathrm{k}-1$, después a la referencia $\mathrm{k}-2$, etc. por lo que la transformación respecto al primer mapa es dado por:

$$
[R, t]_{k \rightarrow 1}=\prod_{i=2}^{k}[R, t]_{i \rightarrow i-1}
$$

donde $[R, t]_{i \rightarrow i-1}$ es la transformación del mapa $\mathrm{i}$ al mapa i-1. 
Rico, J., et al

$$
[R, t]=\left[\begin{array}{ccc}
\cos \left(\theta_{z}\right) & \sin \left(\theta_{z}\right) & T_{x} \\
-\sin \left(\theta_{z}\right) & \cos \left(\theta_{z}\right) & T_{y} \\
0 & 0 & 1
\end{array}\right]
$$

Cada mapa 2D calculado es referenciado al primer mapa utilizando la transformación definida por la ecuación 5, la cual acumula la transformación entre el mapa $\mathrm{k}$ y la primer imagen. Considerando que el desplazamiento del automóvil es en un solo plano, esta transformación es entonces aplicable a la nube de puntos 3D. La nube de puntos adquirida por el LIDAR está referenciada respecto al sistema de referencia del LIDAR, el cual está fijo al automóvil. Nosotros calculamos la posición del automóvil en función del vector de traslación entre los mapas, por otro lado la orientación del automóvil está en función de la rotación de los mapas, ya que se ha calibrado el eje del LIDAR respecto a la referencia del automóvil.

\section{RESULTADOS}

En este trabajo se presenta una aproximación en la construcción de mapas en ambientes estructurados, el ambiente reconstruido representa la plaza Zenea dentro del estado de Querétaro, México. En la propuesta, la extracción del plano de navegación o plano principal es fundamental, tanto para determinar las zonas navegables como la transformación entre cada adquisición. Usando el plano principal como plano de proyección se calcula un mapa 2D usado para navegación y para calcular el desplazamiento del auto entre adquisiciones adyacentes. La acumulación de rotación y traslación entre los planos nos permite calcular la transformación rígida de todos los planos respecto a la primera adquisición para calcular el mapa global 3D y 2D. A partir de la transformación acumulada, nosotros calculamos la posición y orientación del auto.

\section{Construcción de mapas locales}

En la adquisición del LIDAR los datos vienen por paquetes con información de distancias ri, para un ángulo $\theta$ del LIDAR. Cada láser tiene una orientación $\phi \mathrm{i}$ (donde $\mathrm{i}=1,2, \ldots$. 64). La ecuación 1 permite modelizar la normal de un plano cuando coincide con el plano formado por los 64 láseres, si no es el caso, el error de la modelización aumenta. El error de la modelización es representado por el valor propio de la solución a la ecuación 1. Utilizando los datos 3D mostrados en la figura 1 (c). El valor propio más pequeño de la solución de la ecuación 1 está graficada en la figura 4, donde se observa el valor $\theta, \phi$ y h para cada ángulo de rotación del LIDAR. El valor mínimo de los parámetros $\theta$ y $\phi$ corresponden a la orientación de la normal en coordenadas esféricas. El tercer parámetro $h$ corresponde a la distancia perpendicular del plano respecto a la referencia del LIDAR. Con el objetivo de tener una resolución más exacta de los parámetros $\theta, \phi$ y $\mathrm{h}$ se desarrolla una interpolación. Para el valor de $\theta$ los datos cerca del mínimo son aproximados a una parábola $\mathrm{y}$, a partir de la ecuación 1 se calcula el ángulo $\theta$ para un mínimo valor propio. Los datos de $\phi$ vs valor propio son aproximados a una cuadrática, mientras que los de $\mathrm{h}$ a una línea. Los valores de $\phi$ y h son extrapolados cuando el valor del valor propio es cero. Para este caso en particular, a un ángulo de orientación del LIDAR $\theta=$ $130.7^{\circ}$ se obtiene que el ángulo $\phi$ de la normal respecto al eje $\mathrm{Z}$ del LIDAR es de $18.82^{\circ}$ y la distancia del LIDAR al plano principal es de 174.5 centímetros. Con estos datos se segmenta el plano principal. El resultado de la segmentación esta dado en la figura 5 .
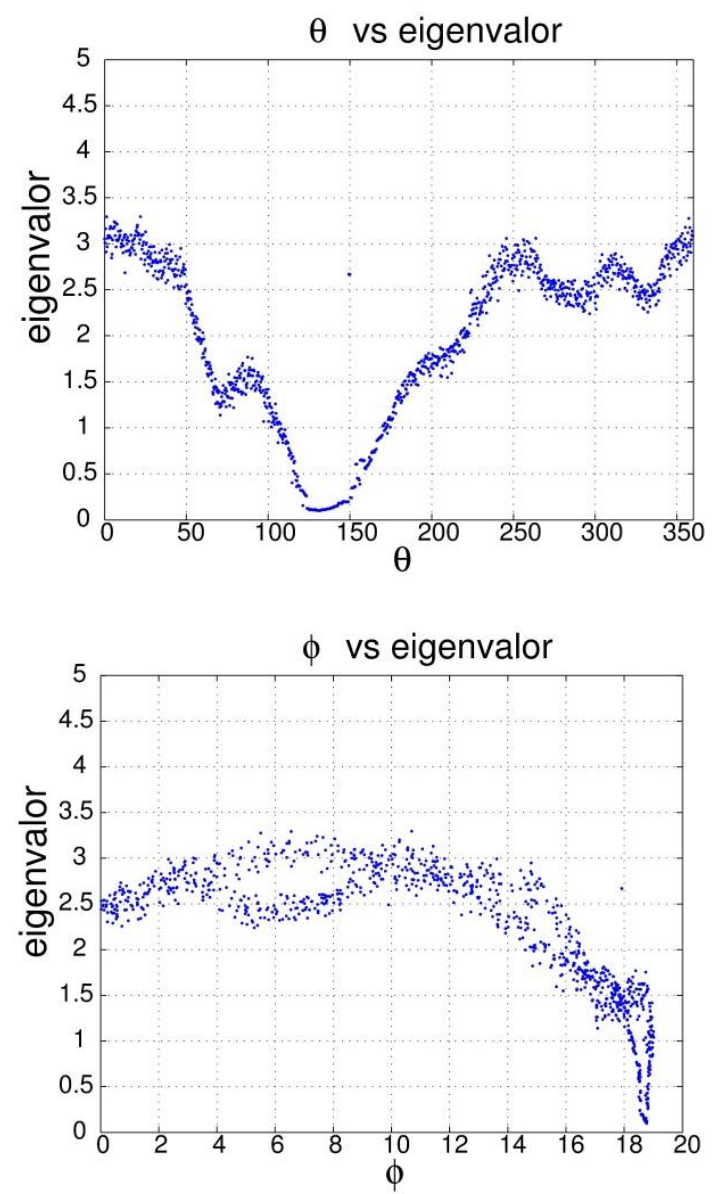
Rico, J., et al

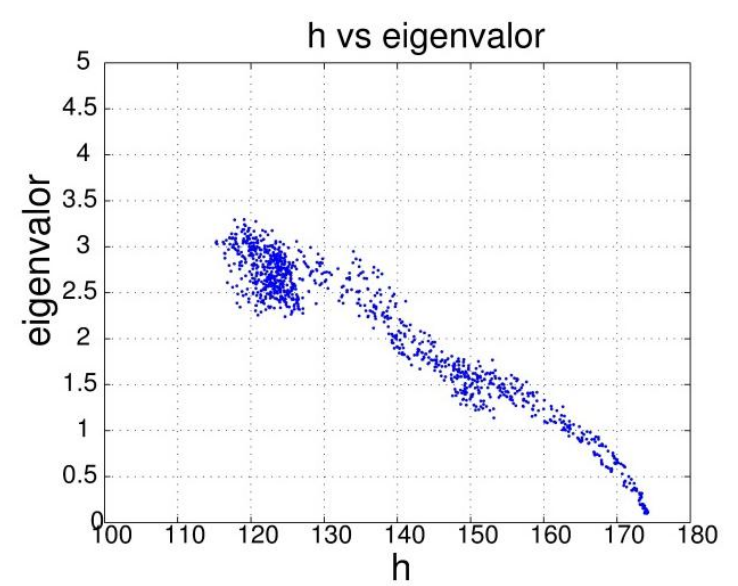

Figura 4. Resultados de $\theta, \phi \mathrm{y}$ h respecto al valor propio $\left(\sigma_{1}\right)$ de la solución de la ecuación 1

La figura 6 muestra el resultado del proceso de creación de los mapas 2D, en el inciso (a) se presenta la proyección en el plano $\mathrm{X}^{\prime} \mathrm{Y}^{\prime}$ del resto de puntos, en los incisos (b) y (c) se muestran dos vistas del mapa MBI , nótese que algunos puntos aislados fueron eliminados utilizando un $\tau=5$ y no fue necesario usar el filtro de eliminación de ruido.

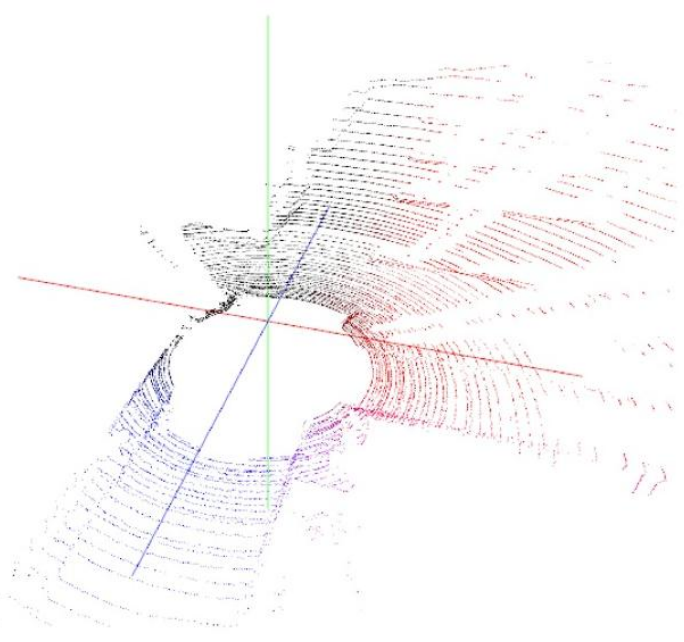

(a) Plano principal

En la figura 7 se muestra una vista tridimensional de la nube de puntos de la plaza Zenea de Querétaro, México ( $20^{\circ} 35^{\prime} 34.82^{\prime \prime} \mathrm{N} 100^{\circ} 23^{\prime} 31,49^{\prime \prime} \mathrm{O}$ ). Un total de 133 adquisiciones fueron tomadas al rededor de la plaza y se usaron para mostrar el resultado de la creación de los mapas 2D y 3D del ambiente recorrido por el automóvil. La figura 8 muestra el mapa 2D de la misma captura que la figura 7 pero ahora creado con los niveles de intensidad con la que regreso el láser (MINT ). Con este mapa se identifican claramente zonas donde hay objetos que regresan una intensidad alta y que son tomados como puntos de interés de manera automática por el detector de Harris \& Stephen.

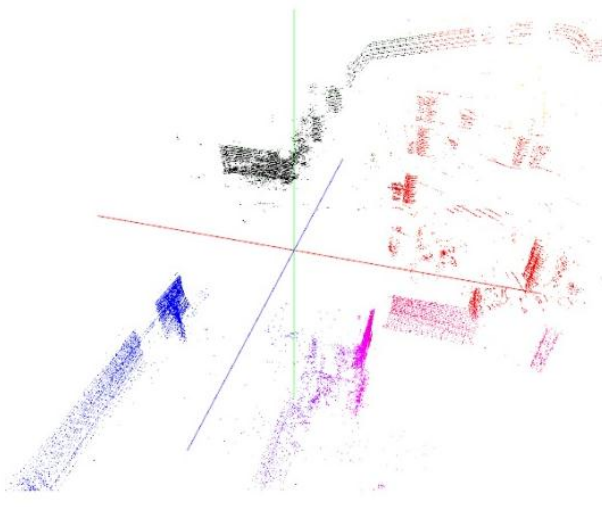

(b) Resto de puntos

Figura 5. Segmentación del plano principal a partir de los datos extrapolados de la figura 4.

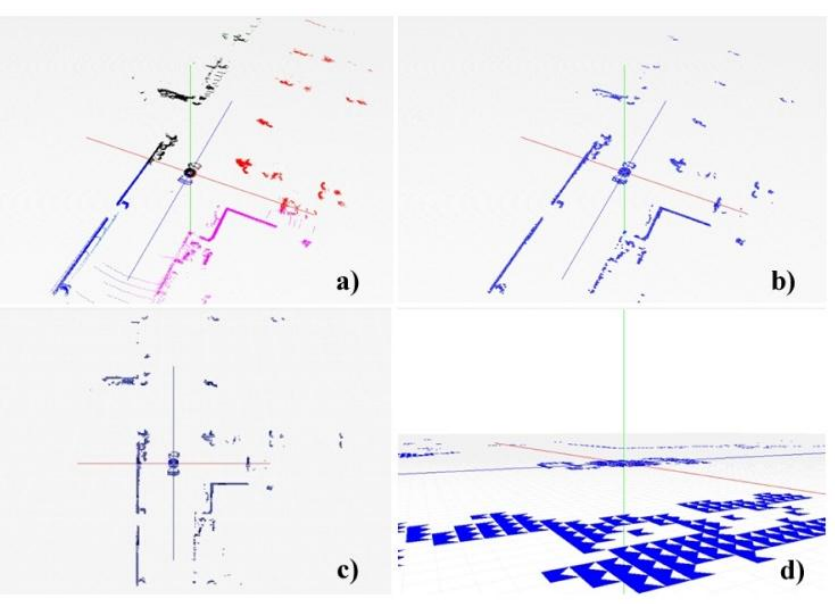

Figura 6. a) Proyección de los puntos en los ejes $X^{\prime}, Y^{\prime}$. b) y c) mapas de navegación $\mathrm{M}_{\mathrm{BI}}$ en diferente perspectiva, d) Mapa de navegación $\mathrm{M}_{\mathrm{BI}}$ ampliado donde las zonas sombreas no son navegable.

\section{Construcción de un mapa global}

La figura 9 muestra los puntos de interés calculado en dos imágenes de intensidad (MINT ) consecutivas y numerados los puntos de correspondencia entre ellas. La correspondencia se hace por correlación de la intensidad de los vecinos de los puntos de interés. Durante el proceso de correspondencia de puntos es común 
encontrar falsos positivos, por ejemplo el punto de interés número 7 de la figura 9 , los cuales son eliminados por las restricciones geométricas dados por la mayoría de los puntos de correspondencia. Los puntos de correspondencia son usados para calcular la matriz transformación afín entre las dos tomas consecutivas.

Se realizó una prueba de campo en la plaza Zenea de Querétaro, México, la cual tiene dimensiones aproximadas de $100 \times 65$ metros. La reconstrucción del mapa 2D se observa en la figura 10. Las posiciones del automóvil en cada adquisición son mostradas por círculos en blanco en la figura 11 (b). Estas posiciones son calculadas a partir de la matriz de transformación afín acumulada. La distancia entre las adquisiciones están representadas en el histograma de la figura 11 (a), en promedio la distancia entre adquisiciones es de aproximadamente 2 metros. Una parte de la reconstrucción $3 \mathrm{D}$ de todo el ambiente se muestra en la figura 12, obsérvese como el punto ciego del LIDAR que aparece en la figura 7 se pierde debido a la superposición de varias tomas a diferentes posiciones.

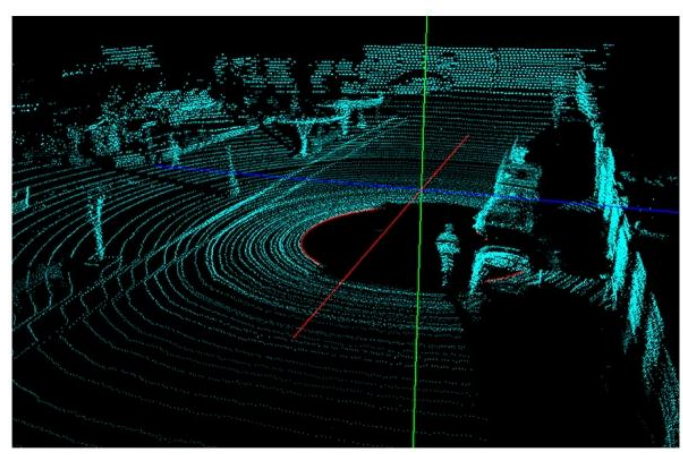

Figura 7. Vista 3D de la nube de puntos de la plaza Zenea de Quéretaro, México (20³5'34.82"N 100²3'31.49"O). Obsérvece un punto sin reconstruir (punto ciego del LIDAR) en el centro de la adquisición. Cerca de este punto se observa una persona y al lado derecho algunos automóviles

En la figura 12 se muestra una nube de puntos que es el resultado de la fusión de las capturas continuas del LIDAR en la plaza Zenea de Querétaro, México, se puede notar claramente el traslape de las líneas circulares que los láseres y como los huecos presentes en una sola toma van desapareciendo.

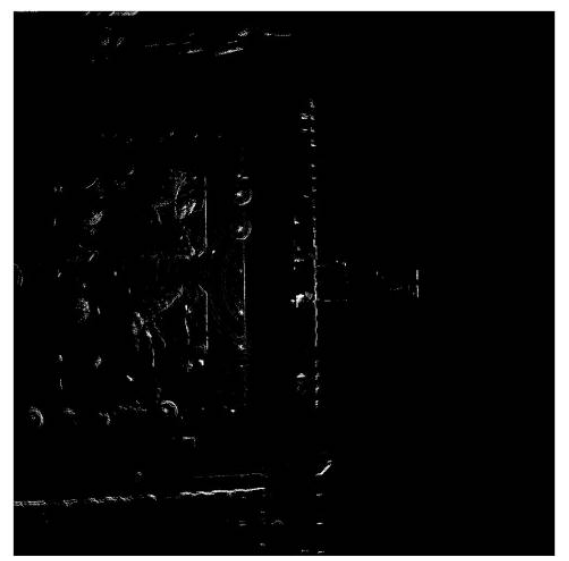

Figura 8. Mapa 2D formado con intensidad del láser $\left(\mathrm{M}_{\mathrm{INT}}\right)$.

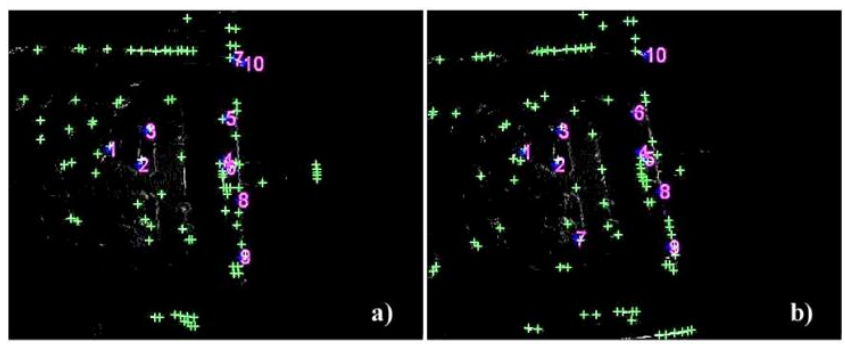

Figura 9. Selección de los puntos clave, a) primer mapa. b) segundo mapa

Los resultados de las figuras 10 y 12 muestran la reconstrucción $2 \mathrm{D}$ y $3 \mathrm{D}$ respectivamente, en las cuales para realizar la correspondencias de mapas locales se tomó en cuenta las transformaciones de rotación y traslación con respecto al sistema de referencia del sensor LIDAR. La superposición de mapas adquiridos en diferentes posiciones permiten definir mejor el ambiente en el cual el automóvil se desplaza y el tamaño del área a reconstruir no está limitado al alcance del LIDAR.

\section{CONCLUSION Y TRABAJOS FUTUROS}

En este trabajo se ha presentado la construcción de mapas 3D y 2D para ambientes estructurados, los mapas globales sirven como base para la navegación autónoma de vehículos. Partiendo de ese conocimiento, hemos considerado que el automóvil se desplaza en un plano. Por tal motivo la transformación entre dos adquisiciones está representada por una traslación en $\mathrm{X}$ e Y así como una rotación sobre el eje $\mathrm{Z}$. 
Rico, J., et al

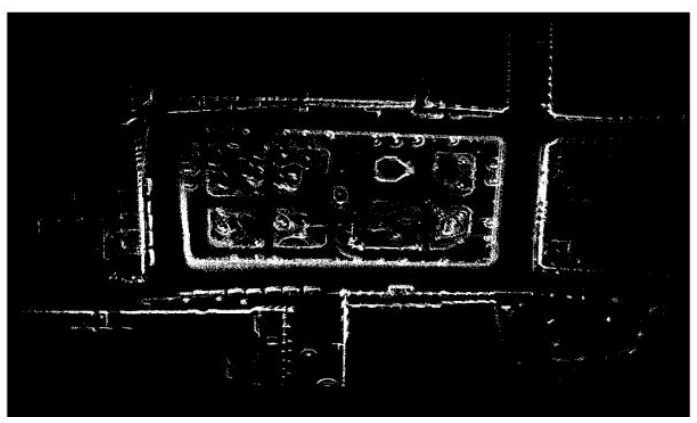

Figura 10. Reconstrucción 2D del mapa de obstáculos de todo el ambiente recorrido por el automóvil

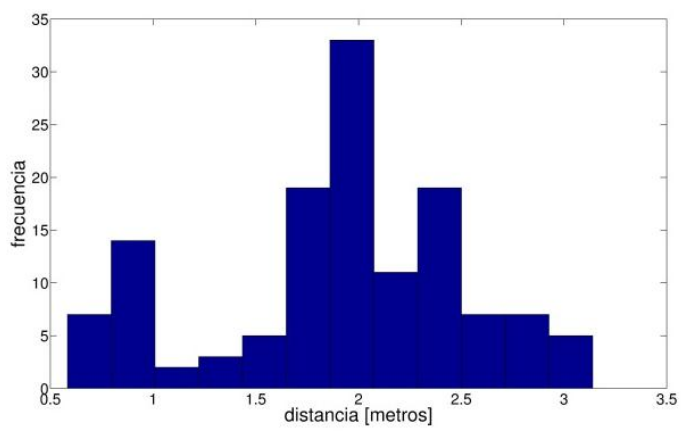

a) Histograma de distancia

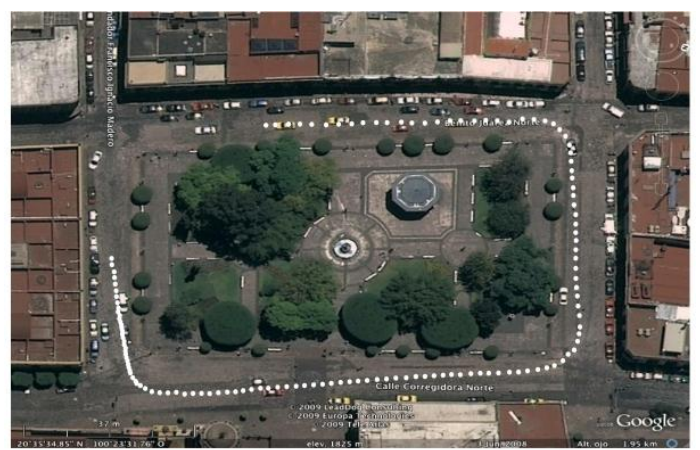

b) Vista aérea de la plaza de Querétaro, México

Figura 11. a) Histograma de distancia entre adquisiciones adyacentes. b) Imagen satelital de la plaza Zenea de Querétaro, México (203'34.82" N 100²3'31.49" O) con las posiciones calculadas del automóvil en círculos blancos. Se presentó que mediante mapas locales se puede calcular transformaciones que ayuden a obtener un mapa global y así obtener la reconstrución de un ambiente exterior más amplio

La reconstrucción 3D de todo el ambiente (ver figura 12) es difícil de interpretar a simple vista, ya que son únicamente puntos 3D. En trabajos futuros, se le incluirá textura a la nube de puntos, para esto se usará una cámara panorámica con un campo de visión horizontal de $360^{\circ}$.

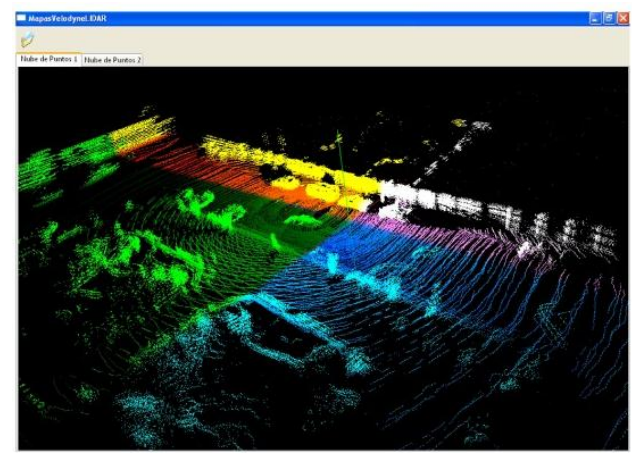

Figura 12. Vista parcial del resultado de la unión de las nubes de puntos $3 D$ del recorrido del automóvil en la plaza Zenea de Querétaro, México.

\section{AGRADECIMIENTOS}

Este trabajo ha sido parcialmente apoyado por CONACYT CB-2005-01-51004/25293 y SIP-IPN 20120472.

\section{REFERENCIAS BIBLIOGRAFICAS}

Arayici, Yusuf (2007). An approach for real world data modelling with the 3 dnext term terrestrial laser scanner for built environment. ISPRS Journal of Photogrammetry and Remote Sensing 16(6), pp. 816829.

Baiely, T. and H. Durran-Whyte (2006). Simultaneous localization and mapping: Part ii. Robotics \& Automation Magazine, IEEE 13(3), pp. 108-117.

Durran-Whyte,H. and T. Bailey (2006). Simultaneous localization and mapping: Part i. Robotics \& Automation Magazine, IEEE 13(2), pp. 99-110.

Frese, U. (2006). A discussion of simultaneous localization and mapping. Autonomous robots 20(1), pp. 25-45.

Harris, C. and M. Stephens (1988). A combined corner and edge detector. 4th Alvey Vision Conference pp. 147-151.

Schleicher D, Bergasa, L.M., Ocana M., Barea R and Lopez M.E. (2009). Real-Time Hierarchical Outdoor SLAM Based on Stereovision and GPS Fusion. Intelligent Transportation Systems, IEEE Transactions on (10) pp. 440-452.

Gil A, Martinez Mozos O, Ballesta M. and Reinoso O. (2010). A comparative evaluation of interest point 
detectors and local descriptors for visual SLAM, Machine vision and Applications (21), pp.905-920.

Lingemann, Kai, Andreas Nuchter and Joachim Hertzberg (2005). High-speed laser localization for mobile robots. Robotics ans Autonomous Systems 51, pp. 275-296.

Mullane, J., J. Ebi, D.Adams and W. W. Sardha (2007). Including probabilistic target detection attributes into map representations. Robot. Auton. Syst. 55(1), pp. $72-$ 85.

Caballero F; Merino L; Ferruz J. and Ollero A. (2009) Vision-Based Odometry and SLAM for Medium and High Altitude Flying UAVs. Unmanned Aircraft Systems, Springer Netherlands, pp. 137-161.

Wangsiripitak, S. and Murray, D.w. (2009). Avoiding moving outliers in visual SLAM by tracking moving objects. Robotics and Automation. ICRA'09. IEEE International Conference on. ISSN $=1050-4729$, pp. 375-380.

$\mathrm{Pu}$, Shi and George Vosselman (2009). Knowledge based reconstruction of building models from terrestrial laser scanning data. ISPRS Journal of Photogrammetry and RemoteSensing, v.64, ISSN 0924-2716. pp. 575584.

N. Fioraio and K. Konolige (2011). Realtime visual and point cloud slam. In Proc. Of the RGB-D Workshop on Advanced Reasoning with Depth Cameras at Robotics: Science and Systems Conf.

Eade Ethan and Drummond Tom (2009). Edge landmarks in monocular SLAM. Image and Vision Computing (27), pp.588-596.

J. J. Rico Jiménez (2009). Construcción de mapas 3D a partir de la extracción de primitivas geométricas obtenidas de datos de una LIDAR. Master's thesis, CICATA-IPN. pp. 27-44.

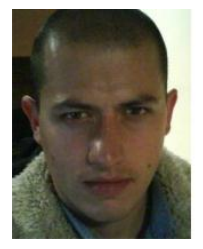

MTA. José de Jesús Rico Jiménez. Estudiante de doctorado en Texas A\&M University (2012) en el área de ingeniería biomédica (EE.UU.), es Maestro en Tecnología Avanzada por CICATA, unidad Querétaro del IPN-

México (2009).

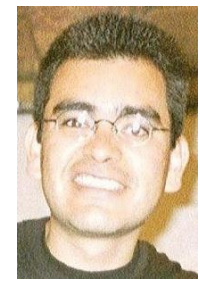

Dr. José Joel González Barbosa. Profesor Investigador del CICATAQuerétaro, estudio Doctorado en Informática y Telecomunicaciones en el Instituto Nacional Politécnico de Toulouse, Francia (2004). estudio Maestría en Informática y Telecomunicaciones el Instituto Nacional Politécnico de Toulouse, Francia (2000), es Maestro en Ingeniería Eléctrica por la Universidad de Guanajuato (1998), es Ingeniero en Electrónica y Telecomunicaciones por la Universidad de Guanajuato (1996).

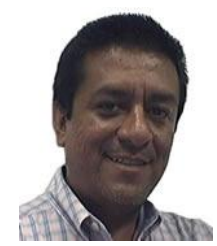

Dr. Juan Bautista Hurtado Ramos. Es profesor investigador, miembro del Sistema Nacional de Investigadores, México. Estudio su Doctorado en el Centro de Investigaciones en Óptica con título expedido por la Universidad de Guanajuato, (1999). Licenciado en Ciencias de las Comunicaciones e ingeniería electrónica (1989) por la Universidad de Guadalajara.

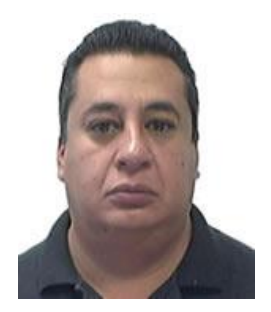

Dr. Francisco Javier Ornelas Rodríguez. Profesor Investigador del CICATA-Querétaro, miembro del Sistema Nacional de Investigadores de México, SNI Nivel I. Doctor en Óptica por el Centro de Investigación en Óptica (1999), Ingeniero en Electrónica (1993) en el Instituto Tecnológico de Celaya, Guanajuato.

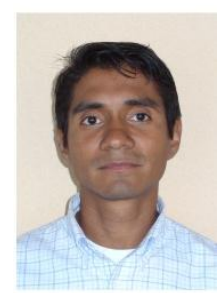

MTA. Denis Eduardo Hernández García. Estudiante de doctorado en Tecnología Avanzada (2012) en CICATA-Querétaro del Instituto Politécnico Nacional, Maestro en Tecnología Avanzada en CICATAQuerétaro del Instituto Politécnico Nacional de México (2011), Ingeniero de Sistemas por la Universidad Nacional de Ingeniería de Nicaragua(2004). Docente Investigador de la FCyS de la Universidad Nacional de Ingeniería de Nicaragua.

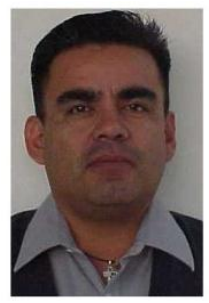

MSC. Ricardo González Barbosa. Profesor Investigador de la Universidad de Guanajuato, es Maestro en Ciencias Ambientales en la línea de Calidad en aguas subterráneas por la Universidad de Irapuato-Salamanca, Ingeniero Químico, es maestro de estudios de Riego en la Universidad de Guanajuato. 